\title{
Utilizing Principal Singular Vectors for 2D DOA Estimation in Single Snapshot Case with Uniform Rectangular Array
}

\author{
Yuntao Wu, ${ }^{1}$ Xiaobing Pei, ${ }^{2}$ and Hing Cheung So ${ }^{3}$ \\ ${ }^{1}$ Key Lab for Intelligent Robot in Hubei Province, Wuhan Institute of Technology, Wuhan 430073, China \\ ${ }^{2}$ School of Software, Huazhong University of Science and Technology, Wuhan 430074, China \\ ${ }^{3}$ Department of Electronic Engineering, City University of Hong Kong, Kowloon Tong, Hong Kong
}

Correspondence should be addressed to Yuntao Wu; ytwu@sina.com

Received 18 February 2015; Accepted 4 May 2015

Academic Editor: Atsushi Mase

Copyright (C) 2015 Yuntao Wu et al. This is an open access article distributed under the Creative Commons Attribution License, which permits unrestricted use, distribution, and reproduction in any medium, provided the original work is properly cited.

\begin{abstract}
The problem of azimuth and elevation directions of arrival (DOAs) estimation using a uniform rectangular array (URA) in single snapshot case is addressed in this paper. Using the principal singular vectors of the observed data matrix, an iterative procedure based on the linear prediction property, and weighted least squares is proposed for finding the DOAs with lower computational complexity. Furthermore, the azimuth and elevation parameters are automatically paired. Computer simulations are included to demonstrate the effectiveness of the proposed algorithm.
\end{abstract}

\section{Introduction}

Two-dimensional (2D) DOA estimation of multiple narrowband signals is an active research topic in array signal processing due to its wide applications in radar, sonar, radio astronomy, and mobile communications and so forth. In recent years, many high-resolution subspace-based algorithms such as 2D MUSIC- [1] or 2D ESPRIT-based [2,3] as well as the modified unitary ESPRIT algorithms [4] have been proposed to jointly estimate the $2 \mathrm{D}$ parameters with various array geometries. These algorithms are effective in certain conditions; however, high-dimension data stacking and eigendecomposition of a constructed covariance of array output are needed, corresponding to high computational load. To reduce the computational complexity of eigendecomposition, fast algorithms have been reported [5, 6]. In general, a direction-finding algorithm in real application must provide a real-time solution that is computationally efficient and uses only a few array snapshots. In some real scenarios, such as fast time-variant channel in mobile communications and sonar signal processing due to physical constraints, only one or a few snapshots are available. Therefore, the problem of parameter estimation in the case of small number of samples has been addressed in the literature [7-9], where the advanced concept of compressed sensing (CS) is applied. In particular, in the worst case such as automotive radar systems [10], only a single snapshot is available for parameter estimation of multiple spatial sources. That is to say, the problem of single snapshot DOA estimation is important in certain application and corresponding DOA estimation algorithms in singlesnapshot case have been recently proposed [11-17].

In this paper, an algorithm of joint estimation of the azimuth and elevation angles using a uniform rectangular array (URA) is proposed for the case of single snapshot. The main idea is based on the principal-singular-vector utilization for model analysis (PUMA) [18, 19]. With the use of the PUMA technique, not only the estimation performance in single snapshot case is improved but the computational complexity of the proposed method is also largely reduced. Moreover, the 2D parameters are automatically paired. Simulation results show that the proposed method provides better performance but with largely reduced computational load compared with the existing 2D ESPRIT method.

The rest of the paper is organized as follows. The problem formulation is given in Section 1. In Section 2, the proposed 2D DOA estimator for single snapshot case with a URA array is devised. Simulation results are included in Section 3 to evaluate the performance of the proposed method by 


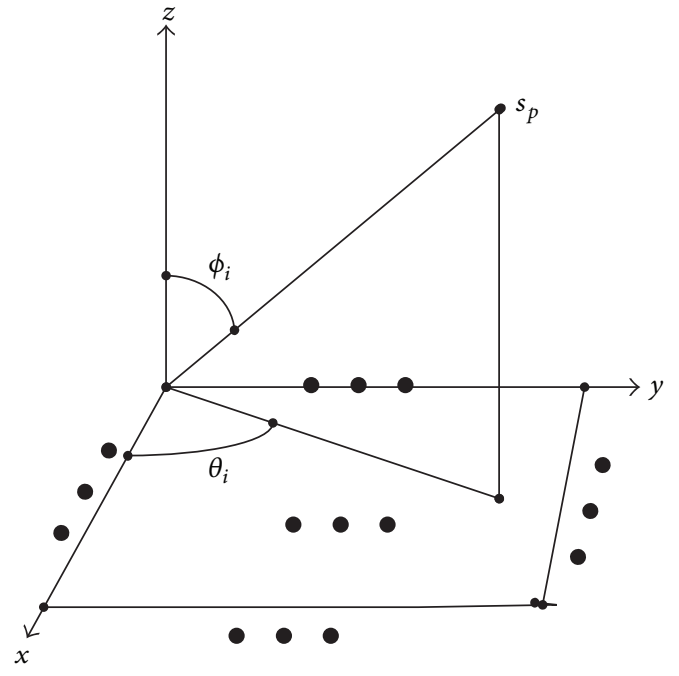

FIGURE 1: Uniform rectangular array geometry.

comparing with the 2D ESPRIT method as well as the corresponding Cramer-Rao lower bound (CRLB). Finally, conclusions are drawn in Section 4.

\section{Problem Formulation}

Figure 1 shows the planar URA configuration for receiving the incoming signals. The array is composed of $M \times N$ omnidirectional antennas with interelement spacing $d$ and we assume that $P$ narrowband signal sources imping on this array from the $2 \mathrm{D}$ spatial directions $\left(\phi_{p}, \theta_{p}\right), p=1,2, \ldots, P$. The sensor located at $a_{11}(1,1)$ is the reference sensor.

The single-snapshot output of the sensor at $(m, n)$ can be expressed as

$$
\begin{aligned}
& x_{m, n} \\
& \quad=\sum_{p=1}^{P} s_{p} e^{j(2 \pi / \lambda)(n-1) d \sin \left(\phi_{p}\right) \cos \left(\theta_{p}\right)} e^{j(2 \pi / \lambda)(m-1) d \sin \left(\phi_{p}\right) \sin \left(\theta_{p}\right)} \\
& +n_{m, n} \quad m=1,2, \ldots, M, n=1,2, \ldots, N,
\end{aligned}
$$

where $s_{p}$ denotes the wavefront of the $p$ th signal source, $\lambda$ denotes the wavelength of the impinging wavefront, and $n_{m, n}$ is the white Gaussian noise with power $\sigma_{n}^{2}$ received at the $(m, n)$ th sensor.

Our task is to estimate the parameter pairs $\left(\phi_{p}, \theta_{p}\right), p=$ $1, \ldots, P$, from the received array output.

\section{Proposed Method}

In this section, the PUMA algorithm [18] is exploited to estimate the 2D DOA parameters of multiple narrowband sources with URA in the case of single snapshot. Define the spatial frequencies $u_{p}, v_{p}$ as $u_{p}=(2 \pi / \lambda) d \sin \left(\phi_{p}\right) \cos \left(\theta_{p}\right)$ and $v_{p}=(2 \pi / \lambda) d \sin \left(\phi_{p}\right) \sin \left(\theta_{p}\right)$; we first express (1) in matrix form as

$$
\mathbf{X}=\mathbf{S}+\mathbf{N}
$$

where $[\mathbf{X}]_{m, n}=x_{m, n},[\mathbf{S}]_{m, n}=s_{m, n}$, and $[\mathbf{N}]_{m, n}=n_{m, n}$.

The $s_{m, n}$ has the form of

$$
s_{m, n}=\sum_{p=1}^{P} s_{p} e^{j\left((m-1) u_{p}+(n-1) v_{p}\right)} .
$$

It is easy to find that $\mathbf{S}$ can be factorized as

$$
\mathbf{S}=\mathbf{G} \boldsymbol{\Sigma} \mathbf{H}^{T},
$$

where

$$
\begin{aligned}
\mathbf{G} & =\left[\begin{array}{llll}
\mathbf{g}_{1} & \mathbf{g}_{2} & \cdots & \mathbf{g}_{P}
\end{array}\right] \\
\mathbf{\Sigma} & =\operatorname{diag}\left(\left[\begin{array}{llll}
s_{1} & s_{2} & \cdots & s_{P}
\end{array}\right]\right), \\
\mathbf{H} & =\left[\begin{array}{llll}
\mathbf{h}_{1} & \mathbf{h}_{2} & \cdots & \mathbf{h}_{P}
\end{array}\right] \\
\mathbf{g}_{p} & =\left[\begin{array}{llll}
u_{p} & u_{p}^{2} & \cdots & u_{p}^{M}
\end{array}\right]^{T} \\
\mathbf{h}_{p} & =\left[\begin{array}{llll}
v_{p} & v_{p}^{2} & \cdots & v_{p}^{N}
\end{array}\right]^{T}
\end{aligned}
$$

Here, $(\cdot)^{T}$ denotes the transpose operator and $\operatorname{diag}(\mathbf{x})$ is a diagonal matrix with vector $\mathbf{x}$ as its main diagonal. It is seen from (8)-(9) that the elements of $\mathbf{g}_{p}$ and $\mathbf{h}_{p}$ satisfy the linear prediction (LP) relations of $\left[\mathbf{g}_{p}\right]_{k+1}=u_{p}\left[\mathbf{g}_{p}\right]_{k}$ and $\left[\mathbf{h}_{p}\right]_{k+1}=$ $v_{p}\left[\mathbf{h}_{p}\right]_{k}$, where $[\cdot]_{k}$ stands for the $k$ th element of $[\cdot]$.

On the other hand, $\mathbf{X}$ can be decomposed using singular value decomposition (SVD) as

$$
\mathbf{X}=\mathbf{U} \boldsymbol{\Lambda} \mathbf{V}^{H}
$$

where $\mathbf{U}$ and $\mathbf{V}$ contain the corresponding left and right singular vectors, respectively, $\boldsymbol{\Lambda}$ is the diagonal matrix of singular values sorted in descending order, and $(\cdot)^{H}$ stands for the conjugate transpose.

From the decomposition in (4)-(10), we have $\mathbf{S}, \operatorname{rank}(\mathbf{S})=$ $P$, and thus the best rank- $P$ approximation of $\mathbf{S}$ according to (10), denoted by $\widehat{\mathbf{S}}$, is

$$
\widehat{\mathbf{S}}=\mathbf{U}_{s} \boldsymbol{\Lambda}_{s} \mathbf{V}_{s}^{H}
$$

where

$$
\begin{aligned}
\mathbf{U}_{s} & =\left[\begin{array}{llll}
\mathbf{u}_{1} & \mathbf{u}_{2} & \cdots & \mathbf{u}_{P}
\end{array}\right], \\
\mathbf{\Sigma} & =\operatorname{diag}\left(\left[\begin{array}{llll}
\lambda_{1} & \lambda_{2} & \cdots & \lambda_{P}
\end{array}\right]\right), \\
\mathbf{V}_{s} & =\left[\begin{array}{llll}
\mathbf{v}_{1} & \mathbf{v}_{2} & \cdots & \mathbf{v}_{P}
\end{array}\right]
\end{aligned}
$$

are the corresponding signal subspace components.

Since $\mathbf{G}$ and $\mathbf{U}_{s}$ have the same subspace, we have

$$
\mathbf{U}_{s}=\mathbf{G} \mathbf{T}_{1} \text {, }
$$

where $\mathbf{T}_{1}$ is an unknown nonsingular matrix with dimension $P \times P$. 
For each $\widehat{\mathbf{u}}_{p}$, we have the following LP property:

$$
\begin{aligned}
\sum_{l=1}^{P} \alpha_{l}\left[\widehat{\mathbf{u}}_{p}\right]_{m-l} & =0, \\
\alpha_{0} & =1, p=1,2, \ldots, P, m=P+1, \ldots, M,
\end{aligned}
$$

where $\alpha_{l}$ are the LP coefficients. The spatial frequencies $u_{p}$ are related to the following polynomial:

$$
\sum_{l=1}^{P} \alpha_{l} z^{P-l}=0
$$

whose roots are $z=e^{j u_{p}}, p=1,2, \ldots, P$. According to (14), the LP error vector e can be constructed by

$$
\mathbf{e}=\mathbf{D b}-\mathbf{f},
$$

where

$$
\begin{aligned}
& \mathbf{D}=\left[\begin{array}{llll}
\mathbf{D}_{1}^{T} & \mathbf{D}_{2}^{T} & \cdots & \mathbf{D}_{P}^{T}
\end{array}\right]^{T}, \\
& \mathbf{f}=\left[\begin{array}{llll}
\mathbf{f}_{1}^{T} & \mathbf{f}_{2}^{T} & \cdots & \mathbf{f}_{P}^{T}
\end{array}\right]^{T}, \\
& \mathbf{b}=\left[\begin{array}{llll}
\alpha_{1} & \alpha_{2} & \cdots & \alpha_{P}
\end{array}\right]^{T}, \\
& \mathbf{D}_{p}=\left(\begin{array}{cccc}
{\left[\mathbf{u}_{p}\right]_{P}} & {\left[\mathbf{u}_{p}\right]_{P-1}} & \cdots & {\left[\mathbf{u}_{p}\right]_{1}} \\
{\left[\mathbf{u}_{p}\right]_{P+1}} & {\left[\mathbf{u}_{p}\right]_{P}} & \cdots & {\left[\mathbf{u}_{p}\right]_{2}} \\
\vdots & \vdots & \ddots & \vdots \\
{\left[\mathbf{u}_{p}\right]_{M-1}} & {\left[\mathbf{u}_{p}\right]_{M-2}} & \cdots & {\left[\mathbf{u}_{p}\right]_{M-P}}
\end{array}\right) \text {, } \\
& \mathbf{f}_{p}=-\left[\left[\mathbf{u}_{p}\right]_{P+1}\left[\mathbf{u}_{p}\right]_{P+2} \cdots\left[\mathbf{u}_{p}\right]_{M}\right]^{T}, \\
& p=1,2, \ldots, P \text {. }
\end{aligned}
$$

Let $\mathbf{W}$ be the symmetric weighting matrix. The weighted least squares (WLS) estimate of $\mathbf{b}$, denoted by $\widehat{\mathbf{b}}$, is

$$
\widehat{\mathbf{b}}=\arg \min _{\mathbf{b}} \mathbf{e}^{H} \mathbf{W e}=\frac{\mathbf{D}^{H} \mathbf{W f}}{\mathbf{D}^{H} \mathbf{W D}} .
$$

Defining a Toeplitz matrix $\mathbf{C}=\operatorname{Toeplitz}\left(\left[\begin{array}{ll}b_{P} & \mathbf{0}_{1 \times(M-P-1)}\end{array}\right]^{T}\right.$, $\left.\left[\begin{array}{llllll}b_{P} & b_{P-1} & \cdots & b_{1} & 1 & \mathbf{0}_{1 \times(M-P-1)}\end{array}\right]\right)$, where $\mathbf{0}_{1 \times(M-P-1)}$ is a zero matrix with dimension $1 \times(M-P-1)$ and $\mathbf{u}_{s}=$ $\left[\begin{array}{llll}\mathbf{u}_{1}^{T} & \mathbf{u}_{2}^{T} & \cdots & \mathbf{u}_{P}^{T}\end{array}\right]$, the optimal $\mathbf{W}$ is derived as [19]

$$
\begin{aligned}
\mathbf{W} & =\sigma^{2}\left[E\left\{\mathbf{e} \mathbf{e}^{H}\right\}\right]^{-1}=\sigma^{2}\left[E\left\{\mathbf{C} \mathbf{u}_{s} \mathbf{u}_{s}^{H} \mathbf{C}^{H}\right\}\right]^{-1} \\
& \approx \operatorname{diag}\left(\left[\lambda_{1}^{2}, \lambda_{2}^{2}, \ldots, \lambda_{P}^{2}\right]\right) \otimes\left(\mathbf{C C}^{H}\right)^{-1},
\end{aligned}
$$

where $\otimes$ is the Kronecker product. As (19) depends on the unknown $\mathbf{b}$, we follow [18] to estimate the $\mathbf{b}$ in an iterative manner and the estimation procedure is as follows:

(1) Set $\mathbf{W}=\operatorname{diag}\left(\left[\lambda_{1}^{2}, \lambda_{2}^{2}, \ldots, \lambda_{P}^{2}\right]\right) \otimes \mathbf{I}_{N-P}$, where $\mathbf{I}_{N-P}$ is the identity matrix with dimension $N-P$.
(2) Calculate $\widehat{\mathbf{b}}$ using (18).

(3) Compute an updated version of $\mathbf{W}$ using (19) with $\mathbf{b}=$ $\widehat{b}$.

(4) Repeat Steps (2)-(3) until a stopping criterion is reached.

Substituting $\widehat{\mathbf{b}}=\mathbf{b}$ in (15) and solving for the roots, denoted by $\widehat{r}_{p}, p=1,2 \ldots, P$, we have the spatial frequency estimate $u_{p}:$

$$
\widehat{u}_{p}=\angle\left(\widehat{r}_{p}\right)
$$

where $\angle(\cdot)$ denotes the phase angle of $(\cdot)$. In order to obtain the $2 \mathrm{D}$ parameter pairing in an automatic manner, another estimation method for the parameters $v_{p}, p=1,2, \ldots, P$, is presented as follows.

From (3)-(4), we have

$$
\mathbf{X} \approx \widehat{\mathbf{G}} \mathbf{Q}^{T},
$$

where $\widehat{\mathbf{G}}$ is the estimate of $\mathbf{G}$ which is constructed using $r_{p}=$ $\widehat{r}_{p}$ and

$$
\begin{aligned}
\widehat{\mathbf{Q}}^{T} & =\boldsymbol{\Sigma} \mathbf{H}^{T}=\left[\begin{array}{llll}
\mathbf{q}_{1} & \mathbf{q}_{2} & \cdots & \mathbf{q}_{p}
\end{array}\right]^{T}, \\
\mathbf{q}_{p} & =s_{p} \mathbf{h}_{p} .
\end{aligned}
$$

From (21), the least squares estimate of $\mathbf{Q}$ is

$$
\widehat{\mathbf{Q}}=\mathbf{X}^{T}\left(\widehat{\mathbf{G}}^{\dagger}\right)^{T}
$$

where $(\cdot)^{\dagger}$ denotes the pseudoinverse. Noting that the elements of $\mathbf{q}_{p}$ satisfy the same LP property as in $\mathbf{h}_{p}$, we extract $\widehat{\mathbf{q}}_{p}$ from $\mathbf{Q}$ to construct the equations:

$$
\widehat{\mathbf{q}}_{p, l} c_{p} \approx \widehat{\mathbf{q}}_{p, f},
$$

where $\widehat{\mathbf{q}}_{p, l}=\widehat{\mathbf{q}}_{p}(1: N-1)$ and $\widehat{\mathbf{q}}_{p, f}=\widehat{\mathbf{q}}_{p}(2: N)$, respectively. Following [18], the WLS estimate of $c_{p}$ is computed as

$$
\begin{aligned}
\widehat{c}_{p} & =\min _{c_{p}}\left(\widehat{\mathbf{q}}_{p, l} c_{p}-\widehat{\mathbf{q}}_{p, f}\right)^{H} \boldsymbol{\Phi}\left(\widehat{\mathbf{q}}_{p, l} c_{p}-\widehat{\mathbf{q}}_{p, f}\right) \\
& =\frac{\widehat{\mathbf{q}}_{p, l}^{H} \boldsymbol{\Phi} \widehat{\mathbf{q}}_{p, f}}{\widehat{\mathbf{q}}_{p, l}^{H} \boldsymbol{\Phi} \widehat{\mathbf{q}}_{p, l}}, \quad p=1,2, \ldots, P,
\end{aligned}
$$

where the optimum weighting matrix $\Phi$ has the form

$$
\begin{aligned}
\boldsymbol{\Phi} & =\left[E\left\{\left(\widehat{\mathbf{q}}_{p, l} c_{p}-\widehat{\mathbf{q}}_{p, f}\right)\left(\widehat{\mathbf{q}}_{p, l} c_{p}-\widehat{\mathbf{q}}_{p, f}\right)^{H}\right\}\right]^{-1} \\
& =\left(\mathbf{B}_{p} \mathbf{B}_{p}^{H}\right)^{-1},
\end{aligned}
$$

where

$$
\begin{aligned}
& \mathbf{B}_{p} \\
& \quad=\operatorname{Toeplitz}\left(\left[\begin{array}{ll}
-c_{p} & \mathbf{0}_{1 \times(N-2)}
\end{array}\right]^{T},\left[\begin{array}{lll}
-c_{p} & 1 & \mathbf{0}_{1 \times(N-2)}
\end{array}\right]\right) .
\end{aligned}
$$




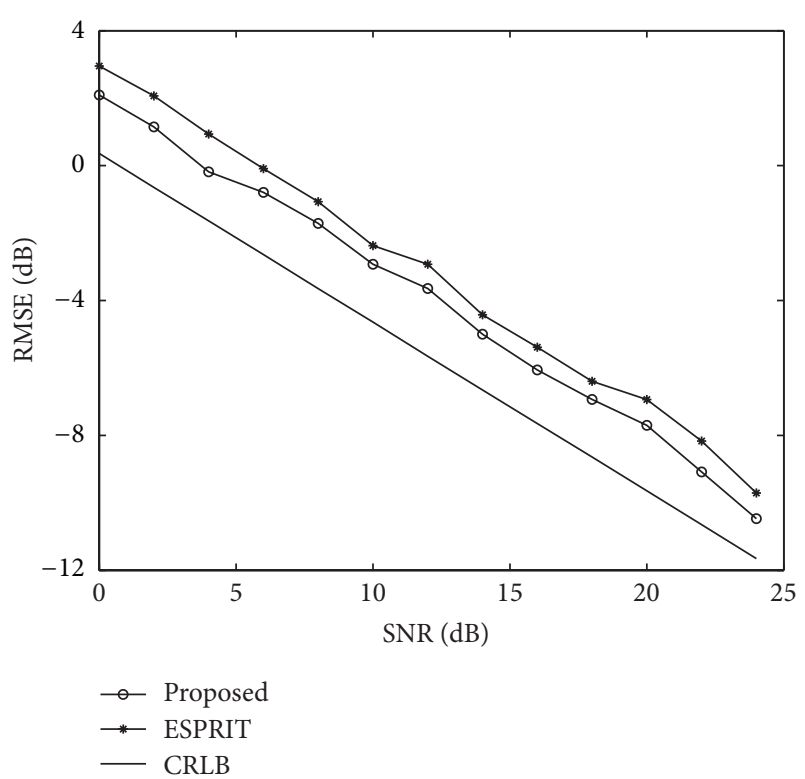

FIgURE 2: Root mean square error for $\phi_{1}$ versus SNR.

Similar to the iterative estimation of $\mathbf{b}$ in finding $u_{p}$, we begin with $\boldsymbol{\Phi}=\mathbf{I}_{N-1}$ in the iterations between (25) and (26) to obtain $\widehat{c}_{p}, p=1,2, \ldots, P$. Finally, the spatial frequencies $v_{p}$, $p=1,2, \ldots, P$, are estimated as

$$
\widehat{v}_{p}=\angle\left(\widehat{c}_{p}\right), \quad p=1,2, \ldots, P .
$$

Note that $\widehat{u}_{p}$ and $\widehat{v}_{p}$ are automatically paired in the whole procedure.

Finally, the estimated azimuth angle $\phi_{p}$ and elevation angle $\theta_{p}$ are computed as

$$
\begin{aligned}
& \widehat{\phi}_{p}=\frac{\lambda}{2 \pi d} \sin ^{-1}\left(\sqrt{\widehat{u}_{p}^{2}+\widehat{v}_{p}^{2}}\right), \\
& \widehat{\theta}_{p}=\tan ^{-1}\left(\frac{\widehat{v}_{p}}{\widehat{u}_{p}}\right), \quad p=1,2, \ldots, P .
\end{aligned}
$$

\section{Simulation Results}

Computer simulations have been conducted to evaluate the 2D DOA estimation performance of the proposed scheme in the presence of white Gaussian noise by comparing with the 2D ESPRIT method [3] and CRLB. The number of iterations in the proposed algorithm is $\mathscr{I}=3$ and the initial parameter estimates are provided by the ESPRIT algorithm [3]. Note that the larger values for $\mathscr{I}$ have been tried but no significant improvement is observed. The number of sensors in the URA is 180 where $M=15$ and $N=12$. Assume that two narrowband signals imping on this received array from the directional angles $\left[\phi_{1}, \phi_{2}\right]=\left[20^{\circ}, 40^{\circ}\right]$ and $\left[\theta_{1}, \theta_{2}\right]=\left[10^{\circ}, 30^{\circ}\right]$, respectively, while the additive noise is white Gaussian process. All results provided are averages of 200 independent runs.

Figures 2-5 plot the root mean square error (RMSE) performance of the $2 \mathrm{D}$ DOA estimates versus SNR. It is

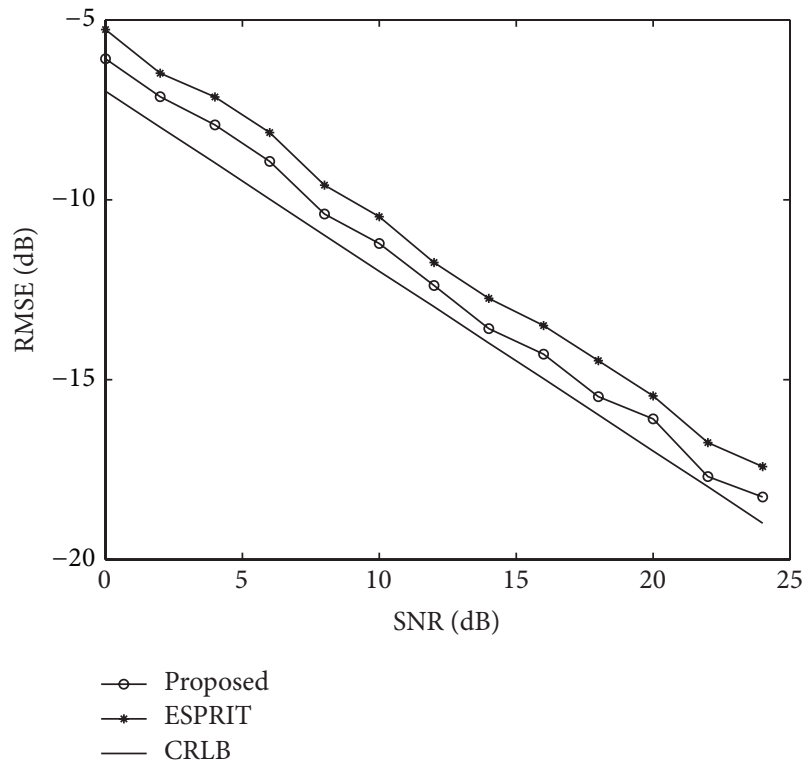

FIgURE 3: Root mean square error for $\theta_{1}$ versus SNR.

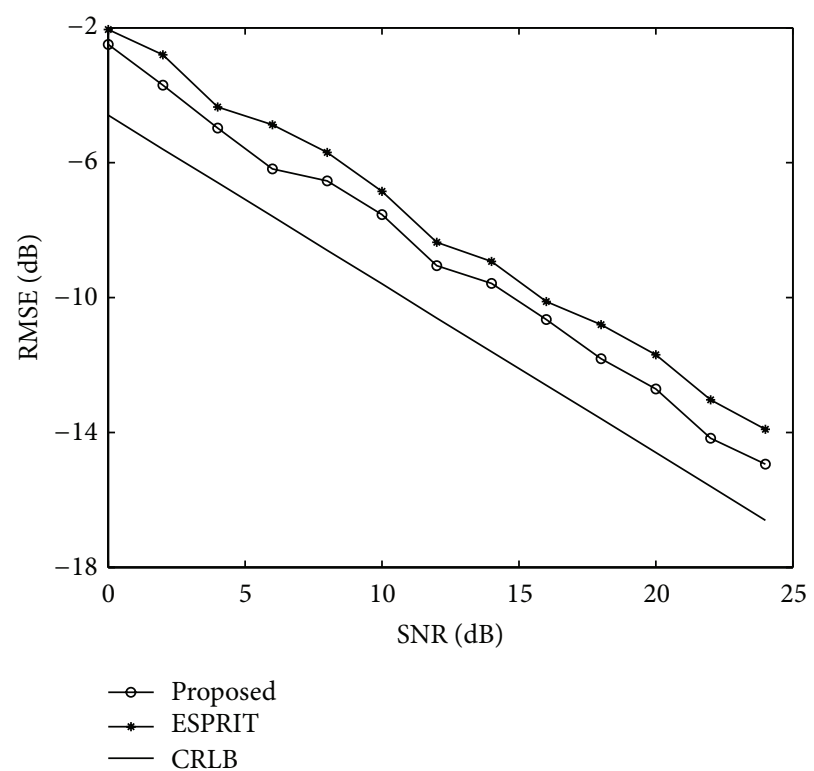

FIGURE 4: Root mean square error for $\phi_{2}$ versus SNR.

seen that the proposed method is superior to the 2D ESPRIT-based method at all signal-to-noise ratio (SNR) conditions. It is because the latter approach is based on the splitting the measurement space into signal subspace and noise subspace and generally gives a higher threshold SNR value. Nevertheless, all the RMSEs of the proposed method are $2 \mathrm{~dB}$ above the corresponding CRLBs at all SNRs. The average computational times of the proposed method and the 2D ESPRIT method are $0.0399 \mathrm{~s}$ and $0.2071 \mathrm{~s}$, respectively, indicating that the former is much more computationally efficient. 


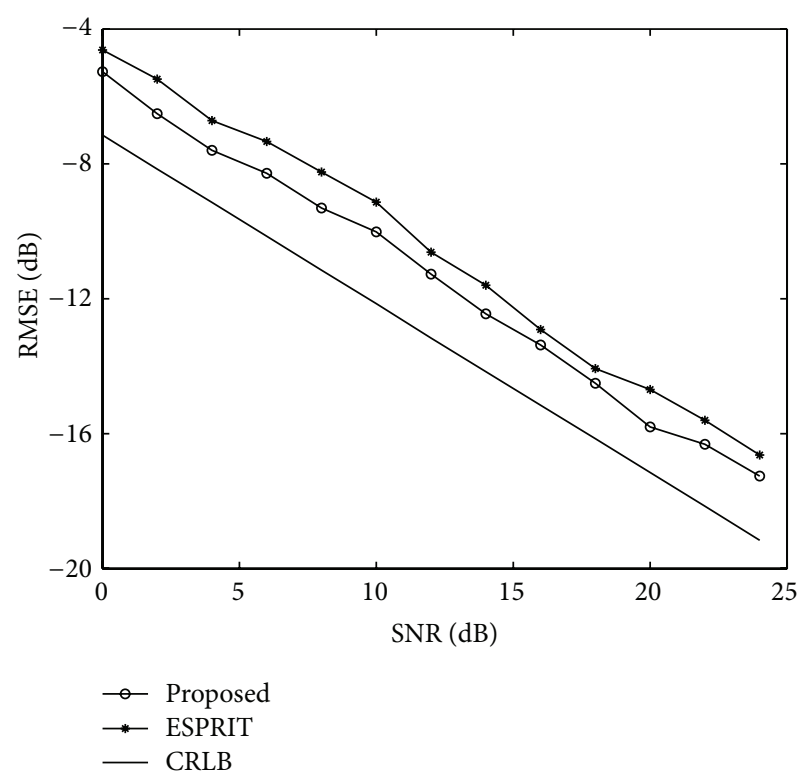

FIgURE 5: Root mean square error for $\theta_{2}$ versus SNR.

\section{Conclusion}

A 2D DOA estimation algorithm with a URA in single snapshot case is proposed using the PUMA method. Compared with the 2D ESPRIT method, the proposed method can obtain higher estimation accuracy and its performance is close to the CRLBs at higher SNR. Moreover, the proposed method shows the advantage of lower computational complexity over the 2D ESPRIT method, and the 2D DOA parameters are automatically paired.

\section{Conflict of Interests}

The authors declare that there is no conflict of interests regarding the publication of this paper.

\section{Acknowledgments}

The work described in this paper was jointly supported by a grant from the National Natural Science Foundation of China (Project no. 61172156), the Program for New Century Excellent Talents in University (NCET-13-0940), the Natural Science Foundation of Hubei Province (no. 2014CFB791), and the Research Plan Project of Hubei Provincial Department of Education (no. T201206).

\section{References}

[1] Y.-M. Chen, J.-H. Lee, and C.-C. Yeh, “Two-dimensional angleof-arrival estimation for uniform planar arrays with sensor position errors," IEE Proceedings Part F: Radar and Signal Processing, vol. 140, no. 1, pp. 37-42, 1993.

[2] R. Roy and T. Kailath, "ESPRIT-estimation of signal parameters via rotational invariance techniques," IEEE Transactions on Acoustics, Speech and Signal Processing, vol. 37, no. 7, pp. 984995, 1989.
[3] T. Kuroda, N. Kikuma, and N. Inagaki, "DOA estimation and pairing method in 2D-ESPRIT using triangular antenna array," Electronics and Communications in Japan, Part I: Communications, vol. 86, no. 6, pp. 59-68, 2003.

[4] M. D. Zoltowski, M. Haardt, and C. P. Mathews, "Closed-form 2-D angle estimation with rectangular arrays in element space or beamspace via unitary ESPRIT," IEEE Transactions on Signal Processing, vol. 44, no. 2, pp. 316-328, 1996.

[5] C.-H. Lin, W.-H. Fang, K.-H. Wu, and J.-D. Lin, "Fast algorithm for joint azimuth and elevation angles, and frequency estimation via hierarchical space-time decomposition," in Proceedings of the IEEE International Conference on Acoustics, Speech and Signal Processing (ICASSP '07), vol. 2, pp. II-1061-II-1064, Honolulu, Hawaii, USA, April 2007.

[6] Y. Wu, G. Liao, and H. C. So, "A fast algorithm for 2-D directionof-arrival estimation," Signal Processing, vol. 83, no. 8, pp. 18271831, 2003.

[7] X. Li, X. C. Ma, S. F. Yan, and C. H. Hou, "Single snapshot DOA estimation by compressive sampling," Applied Acoustics, vol. 74, no. 7, pp. 926-930, 2013.

[8] M. Zhou, X. F. Zhang, X. F. Qiu, and C. H. Wang, "Twodimensional direction of arrival (DOA) estimation for rectangular array via compressive sensing trilinear model," International Journal of Antennas and Propagation. In press.

[9] W. Xiu-hong, M. Xing-peng, and Z. Nai-tong, "Single-snapshot DOA estimation based on compressed sensing in PCR systems," in Proceedings of the IEEE Radar Conference, pp. 528-531, Cincinnati, Ohio, USA, May 2014.

[10] C. J. Huang, C. W. Dai, T. Y. Tsai, W. H. Chung, and T. S. Lee, "A closed-form phasecomparison ML DOA estimation for automotive radar with one single snapshot," IEICE Electron Express, vol. 10, no. 7, pp. 1-7, 2013.

[11] Z. Hui, G. Lindong, and W. Yuexian, "Two-dimension direction finding using single-snapshot data," in Proceedings of the International Conference on Communication Technology (ICCT '06), pp. 1-4, November 2006.

[12] Q. S. Ren and A. J. Willis, "Extending MUSIC to single snapshot and on-line direction finding applications," in Proceedings of the IET International Conference on Radar, pp. 783-787, October 1997.

[13] A. C. Chang and C. C. Shen, "Orthogonal projection DOA estimation with a single snapshot," IEICE Transactions on Communications, vol. E96-B, no. 5, pp. 1215-1217, 2013.

[14] H. Chen and X. Zhang, "Two-dimensional DOA estimation of coherent sources for acoustic vector-sensor array using a single snapshot," Wireless Personal Communications, vol. 72, no. 1, pp. 1-13, 2013.

[15] B. M. Radich and K. M. Buckley, "Single-snapshot DOA estimation and source number detection," IEEE Signal Processing Letters, vol. 4, no. 4, pp. 109-111, 1997.

[16] P. Häcker and B. Yang, "Single snapshot DOA estimation," Advances in Radio Science, vol. 8, pp. 251-256, 2010.

[17] S. Fortunati, R. Grasso, F. Gini, M. S. Greco, and K. LePage, "Single-snapshot DOA estimation by using Compressed Sensing," EURASIP Journal on Advances in Signal Processing, vol. 2014, article 120, 2014.

[18] H. C. So, F. K. Chan, W. H. Lau, and C.-F. Chan, "An efficient approach for two-dimensional parameter estimation of a singletone," IEEE Transactions on Signal Processing, vol. 58, no. 4, pp. 1999-2009, 2010. 
[19] F. K. W. Chan, H. C. So, and W. Sun, "Subspace approach for two-dimensional parameter estimation of multiple damped sinusoids," Signal Processing, vol. 92, no. 9, pp. 2172-2179, 2012. 

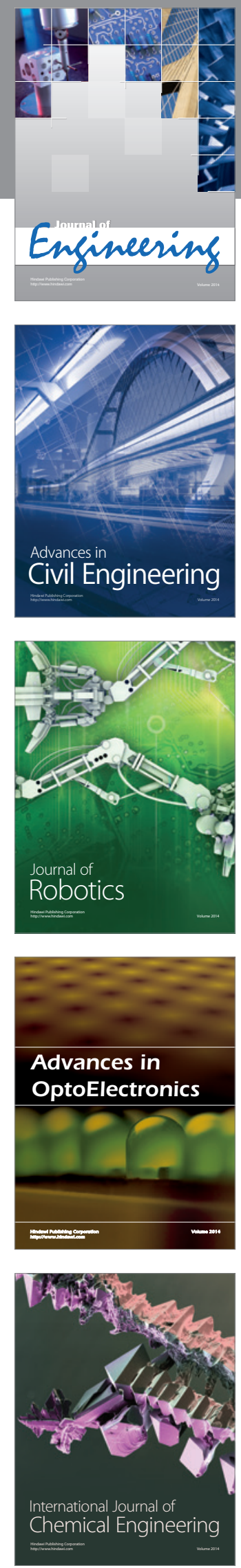

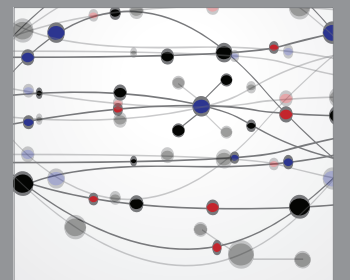

The Scientific World Journal
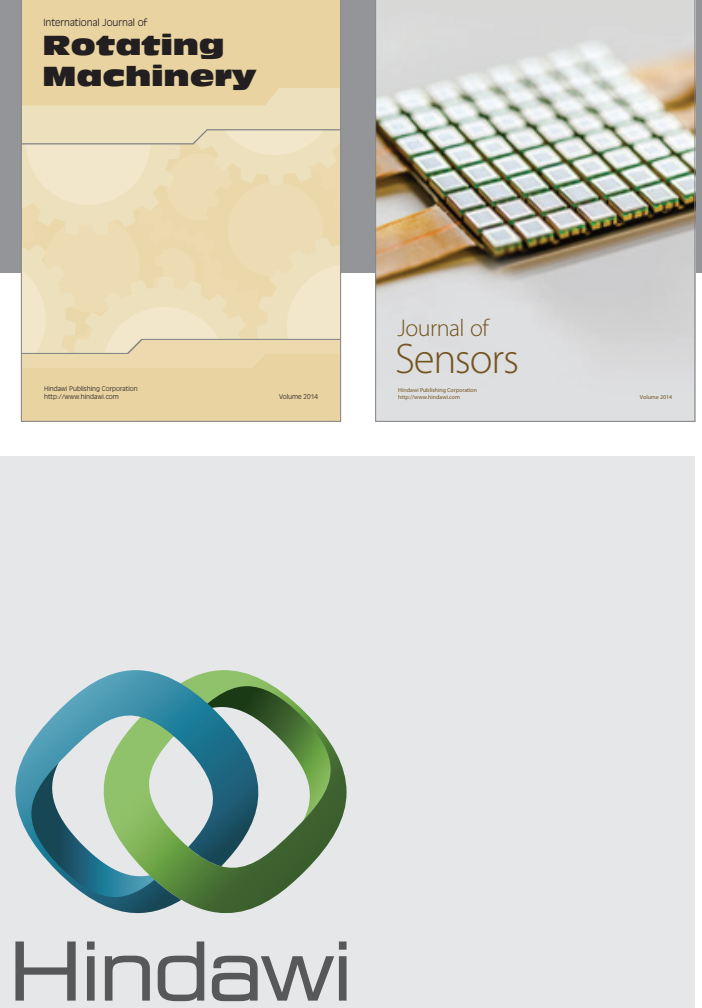

Submit your manuscripts at http://www.hindawi.com
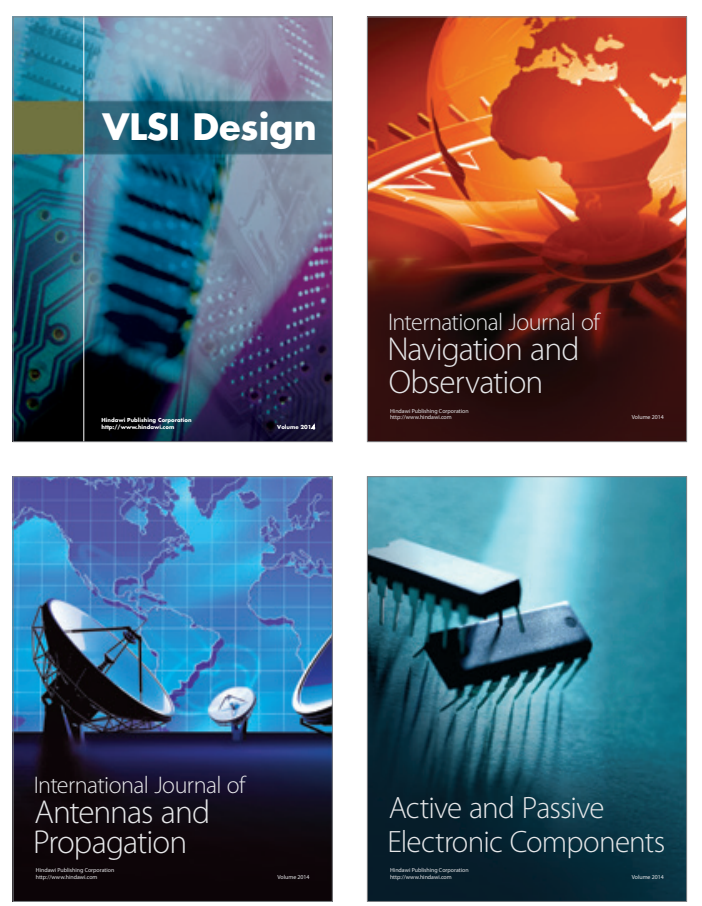
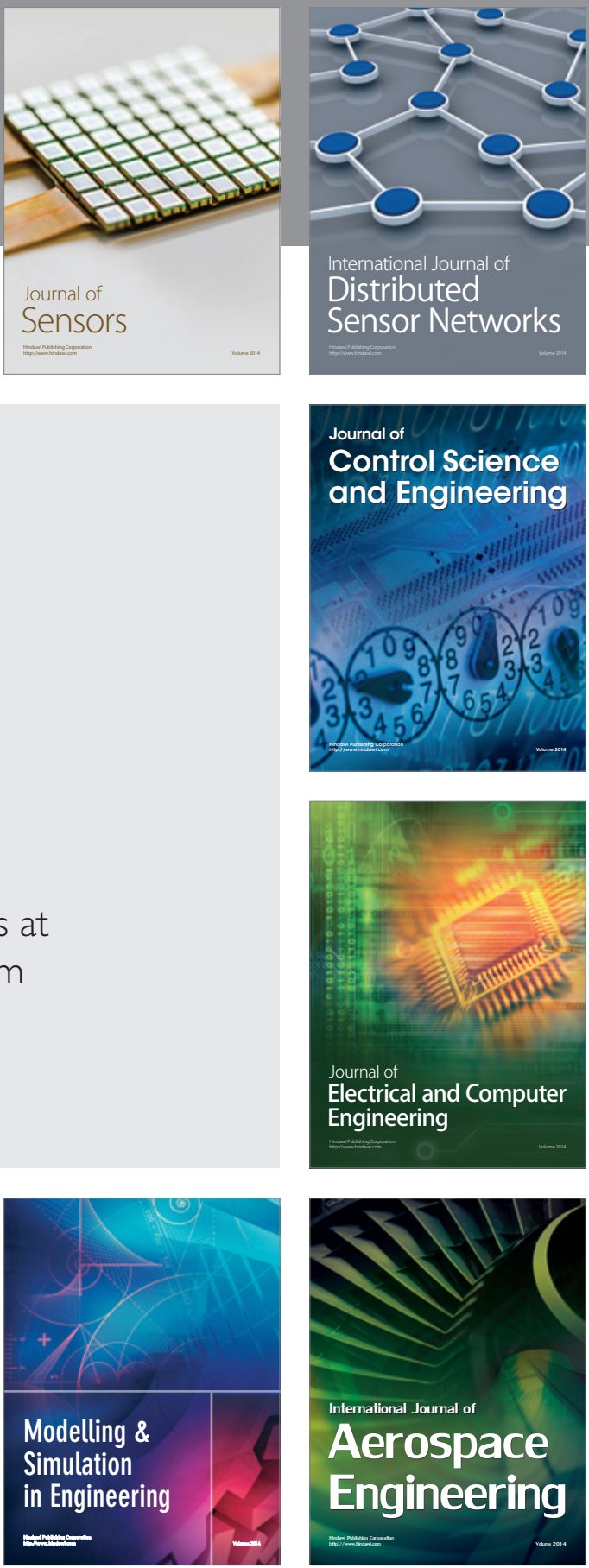

Journal of

Control Science

and Engineering
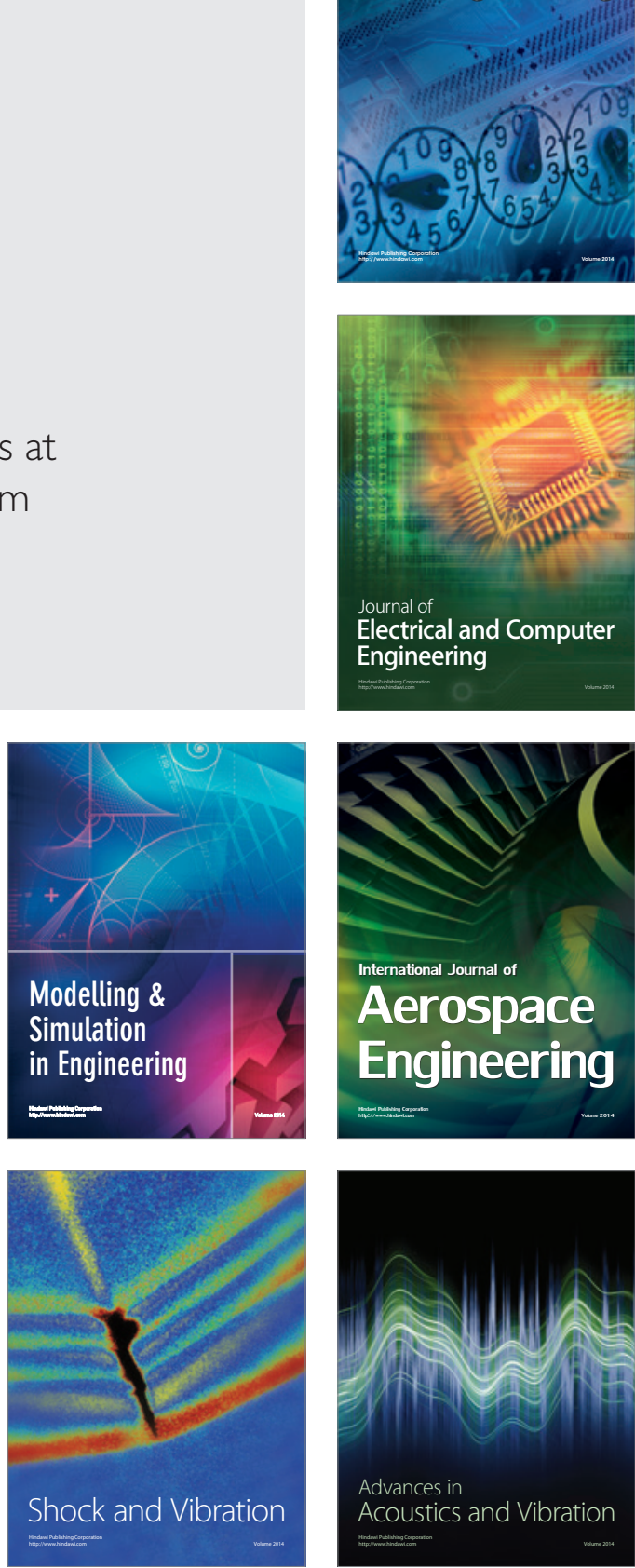